\title{
Multicolored devices fabricated by direct lithography of colloidal nanocrystals
}

\author{
Antonio Qualtieri *, Luigi Martiradonna, Tiziana Stomeo, Maria Teresa Todaro, \\ Roberto Cingolani, Massimo De Vittorio
}

National Nanotechnology Laboratory of CNR/INFM, Scuola Superiore ISUFI, University of Salento, Via per Arnesano, Lecce 73100, Italy

\section{A R T I C L E I N F O}

Article history:

Received 29 September 2008

Received in revised form 12 November 2008

Accepted 14 November 2008

Available online 9 December 2008

\section{Keywords:}

Colloidal nanocrystals

Resist

Photolithography

E-beam lithography

Multicolor display

\begin{abstract}
A B S T R A C T
Colloidal nanocrystals (NCs) are interesting as potential active medium for novel nanophotonic and nanoelectronic devices, due to their low fabrication costs and full tunability of their opto-electronic properties.

In this work we report a multi step approach for the fabrication of multicolored micro- and nano- displays by localizing NCs through lithographic techniques without recurring to etching processes or surface treatment of the substrate.

We demonstrate the possibility to localize different ensembles of emitters on the same substrate by realigned photolithographic steps or mix-and-match electron beam and photolithographic approaches. Moreover, fine tuning of the overall pixel emission is shown by varying the concentration ratio among localized red and green NCs. This technique enables the fabrication of optically pumped colored pixels with very high definition. Our approach combined with advances in NCs-based light emitting diodes technology will enable the electrical injection of advanced micro and nano light sources.
\end{abstract}

(c) 2008 Elsevier B.V. All rights reserved.
In the last years, multicolor and white organic light emitting diodes (OLEDs) aroused the attention of lighting and optoelectronic industry for display applications [1-3]. As compared to inorganic devices based on epitaxial growth [4], organic based technology for light generation is better suited for the fabrication of flexible, large-area panels by low-cost fabrication techniques [5-7]. Thin, flexible, bright camera displays and TV monitors based on OLED technology are nowadays on the market thanks to the joint effort of research labs and industrial companies [8]. Despite the great commercial outcome, still some relevant limitations must be mentioned for organic chromophores: their color emission is not fully saturated as compared to inorganic emitters, and the different aging of red, green and blue (RGB) components used for the fabrication of RGB pixels affects both the overall color stability and the device lifetime [6,7]. On the contrary, semiconductor colloidal nanocrystals (NCs) seem to give an interesting alternative as active materials for novel photonic devices. Similarly to organic emitters, they are synthesized by means of cheap and high-throughput growth facilities, and can be easily deposited through simple techniques such as spin-coating on any, even flexible, substrates. Moreover, they show broader excitation spectra and narrower emission bands, saturated emission tunable from the ultraviolet to the infrared spectral range, and a higher photochemical stability [9-11]. Their increased emission stability and robustness obtained with properly engineered semiconductor shells [12] make them also suited to be embedded in polymeric matrices. The integration of

\footnotetext{
* Corresponding author. Tel.: +39 0832 298316; fax: +39 0832298386

E-mail address: antonio.qualtieri@unile.it (A. Qualtieri).
}

colloidal Quantum Dots (QDs) into organic LEDs [13-16] could lead to an increase of the device lifetimes, and monochromatic and white-emitting QD-LEDs $[15,16]$ have been already demonstrated. In order to realize RGB pixels based on colloidal QDs, patterning techniques able to localize different emitters on the same substrate are required, but very few strategies have been presently proposed [17-19].

Here we show the possibility to realize multicolor patterns by localizing different ensembles of colloidal NCs through lithographic techniques. Direct patterning of inorganic nanoclusters dispersed in polymeric resists, whose polymerization degree can be controlled through exposure to highly energetic ultraviolet or electron beam, revealed to be a simple and precise NCs localization technique $[17,18]$. Positioning of multiple QDs species on the same sample through realigned procedures, here reported, enables the realization of nano- and micro-sized RGB pixels with full tunability of the overall color emission in the visible spectral range, as demonstrated by optical characterization. Once merged with the technological advances achieved in the electrical injection of QD-based LEDs [20,21], this approach could open the way to the fabrication of the next generation of cheap, bright and flexible displays and micro and nano light sources with long operating lifetimes.

In order to understand the influence of concentration and size of NCs on the lithographic properties of the blends, two ensembles of $\mathrm{CdSe} / \mathrm{ZnS}$ core/shell Quantum Dots having different diameters (core diameter $\sim 3.5 \mathrm{~nm}$ for green emitting NCs, $\sim 5 \mathrm{~nm}$ for redemitting NCs) were mixed in solution phase in both poly(methylmethacrylate) (PMMA) [22] and SU-8 epoxy resin [23] (positive and negative resists, respectively). The nanocrystals were prepared 
and diluted in chlorobenzene $\left(\mathrm{C}_{6} \mathrm{H}_{5} \mathrm{Cl}\right)$ by using standard methods $[24,25]$, and characterized with a Cary Eclypse 5 Varian fluorimeter and a Cary 5000 Varian spectrophotometer (their emission and absorption spectra are reported in solid and dashed lines, respectively, in Fig. 1a). The nanocrystals were subsequently dispersed in PMMA and SU-8 with molar concentrations ranging from $\mathrm{M}_{\mathrm{NCs}}=5.0 \times 10^{-7} \mathrm{~mol} / \mathrm{l}$ to $\mathrm{M}_{\mathrm{NCs}}=2.0 \times 10^{-6} \mathrm{~mol} / \mathrm{l}$. The photolumi-

a
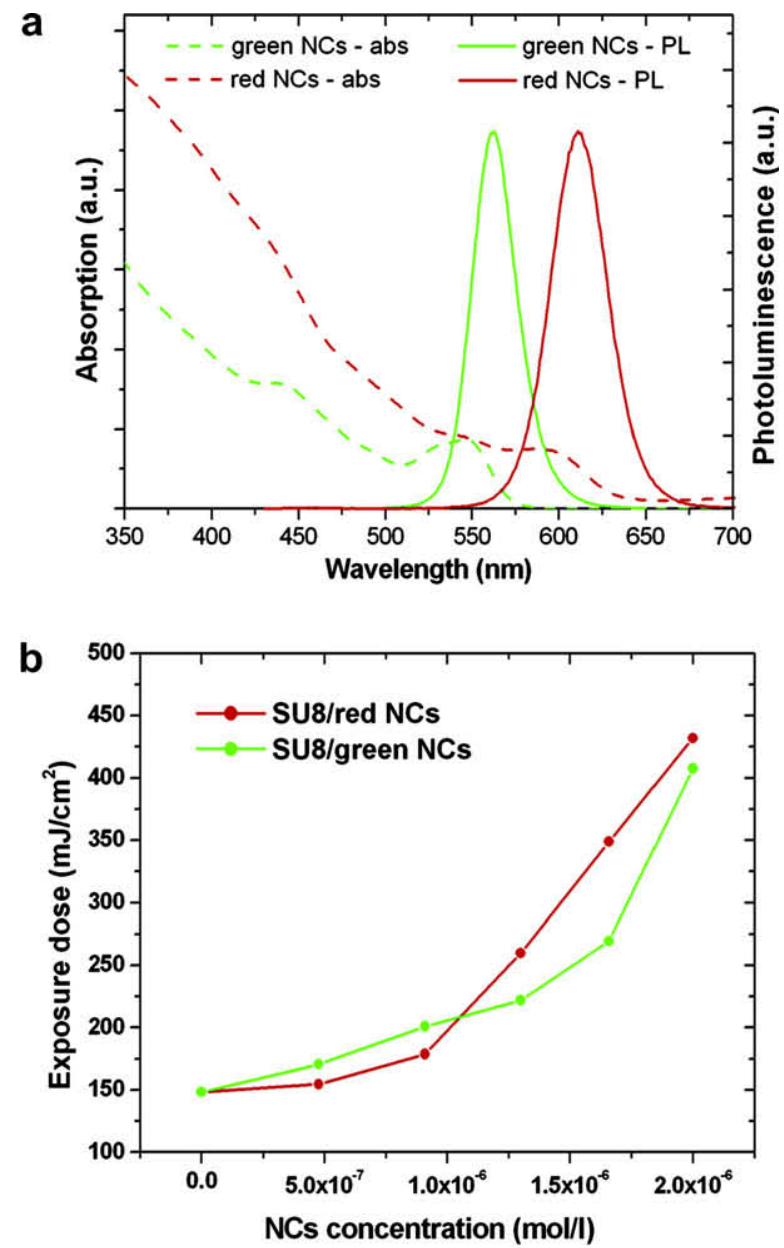

C

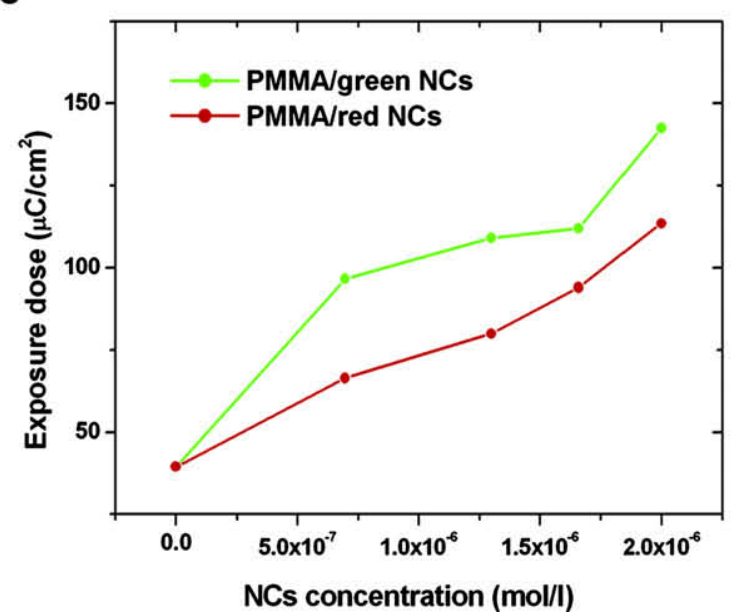

Fig. 1. (a) Emission (solid lines) and absorption (dashed lines) spectra of green (green lines) and red (red lines) emitting CdSe/ZnS NCs diluted in chlorobenzene;

(b) Diagram of exposure dose vs. nanocrystals molar concentration in SU-8; (c) Diagram of exposure dose vs. nanocrystals molar concentration in PMMA. nescence (PL) spectra of the emitters were not significantly modified after their dispersion in resist matrices.

The SU-8 based blends were spin-coated on silicon substrates and exposed to the $\mathrm{Hg}$ i-line $(\lambda=365 \mathrm{~nm})$ of a MJB-3 Karl Suss Mask Aligner. For each concentration, several patterns were realized by using different exposure doses and characterized through scanning electron microscopy (SEM) in order to identify the best exposure conditions. The optimal exposure dose trend (estimated with a precision of $\pm 10 \mathrm{~mJ} / \mathrm{cm}^{2}$ ) was found as reported in Fig. 1b: as expected, it increases as the volume fraction of the semiconductor nanocrystals increases. Notably, the slope of the red-NCs doped SU-8 is higher than the green-NCs doped SU-8 one. This could be due both to the bigger size of the red-emitting QDs which hinders polymer cross-linking and to their higher absorption at $365 \mathrm{~nm}$ (see Fig. 1a).

The PMMA based blends were also spin-coated on silicon substrates and patterned through electron beam lithography, with a RAITH150 lithography system operating at an acceleration voltage of $5 \mathrm{KeV}$. The optimal exposure conditions were estimated similarly to the previous case, with a precision of $\pm 5 \mu \mathrm{C} / \mathrm{cm}^{2}$. A similar increase of the optimal exposure energy with increasing NCs concentration was found, as reported in Fig. 1c, although in this case a linear relationship seems to be followed. The Quantum Dots size seems to influence in an opposite way the sensitivity of this positive e-beam resist as compared to SU-8; we suppose that the different behavior is due to the nanocrystals distribution in the spincoated polymeric layer (as reported in [26]).

In previous works $[18,27]$, we already verified the effectiveness and the outlook of this localization approach. The systematic analysis reported here allows to extend this technique to any kind of colloidal nanocrystals (provided that they are soluble in resist blends) with a broad range of concentrations. Fully-customable micro- and nano- patterns can be therefore obtained by a single lithographic procedure. Importantly, the possibility to re-align subsequent lithographic steps on the same substrate (as schematically shown in Fig. 2a-f) enables the localization of different NCs ensembles in confining or superimposed regions. Thus, this technique can be exploited for the realization of RGB pixels on the micrometer scale, by localizing red, green and blue emitting nanocrystals in contiguous regions. The overall photoluminescence of each pixel results in the sum of the intensities of these chromatic components (as sketched in Fig. 2g). By controlling the molar concentrations of the NCs and by varying the geometrical parameters of the localized patterns, we can finely tune the PL spectrum of these pixels thus obtaining multicolored and white micro and nanoemitters.

In order to demonstrate the potential of this technique, we realized multicolored logos of the National Nanotechnology Laboratory (NNL) by localizing two different ensembles of NCs. A first logo was obtained by exploiting two photolithographic steps: red-emitting nanocrystals were dispersed in SU-8, spin-coated on a Si substrate and exposed to ultraviolet radiation; after development and thermal curing, a second layer of green emitting NCs dispersed in SU8 was deposited on the pattern and exposed. The sample was finally developed and optically characterized by means of an OLYMPUS Fluoview 1000 confocal microscope (Fig. 2h). The NCs were excited with a laser-diode emitting at $405 \mathrm{~nm}$, and two photodetectors were used to collect photoluminescence in two separate spectral ranges (red PL collected in the 630-670 nm spectral range, green PL collected in the $530-570 \mathrm{~nm}$ spectral range). The combined fluorescence map shown in Fig. $2 \mathrm{~h}$ demonstrates that no mixing between the two NCs ensembles can be detected, showing that the localization approach is not affected by the two sequential steps of the lithographic process.

Similarly, a second logo was realized in mix-and-match lithography: a layer of PMMA containing red-emitting nanocrystals was patterned through an e-beam lithography process; SU-8 resist with 


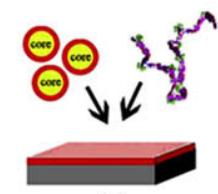

(a)

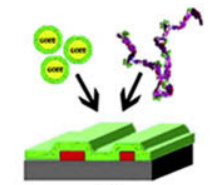

(d)

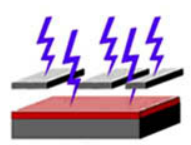

(b)

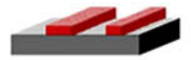

(c)

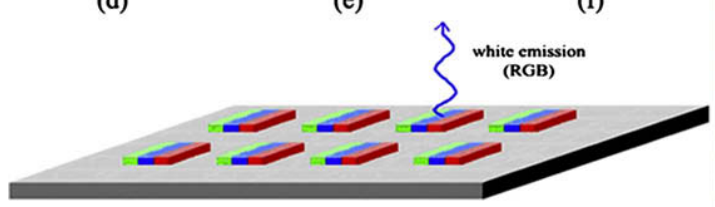

(g)

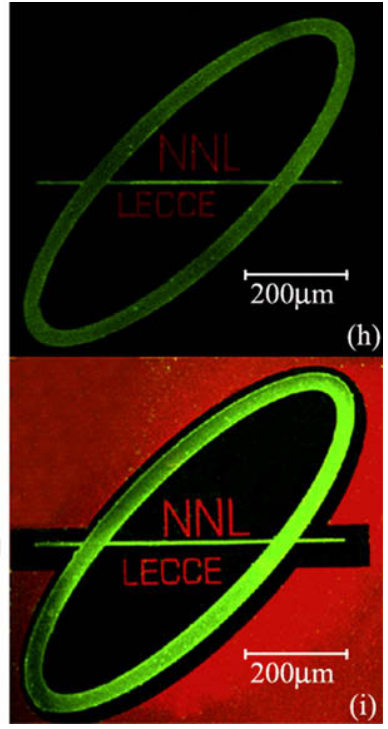

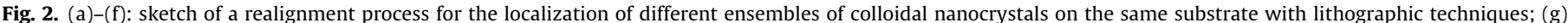

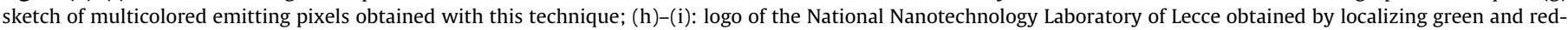
emitting NCs with two photolithographic steps or with a mix-and-match procedure, respectively.

green emitting nanocrystals was then localized on the sample through photolithography. The photoluminescence map of the processed logo is reported in Fig. $2 \mathrm{i}$.
Furthermore, we also verified the possibility to combine the emission of different NCs ensembles by performing localization on superimposed regions. A layer of red NCs/PMMA blend

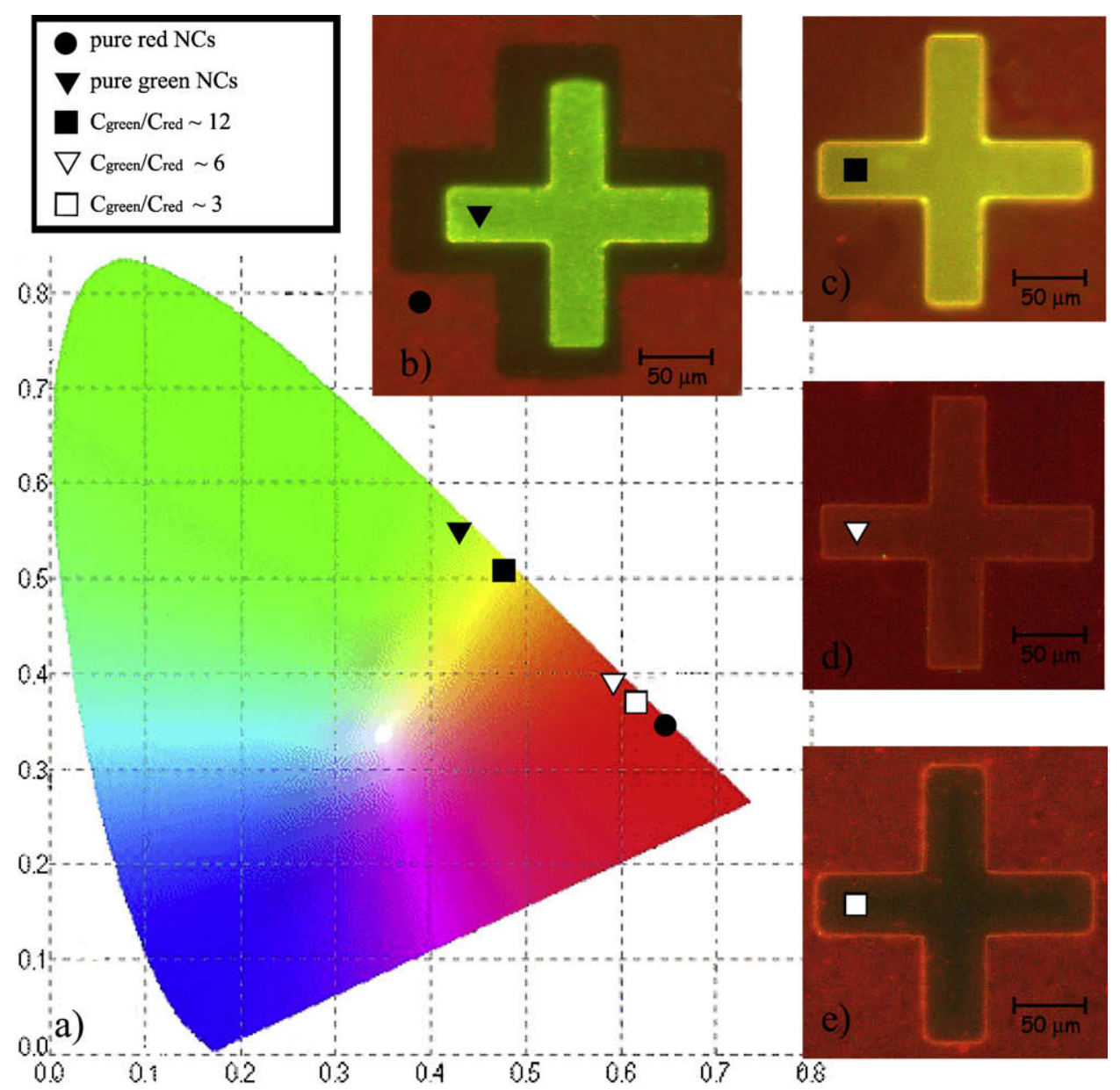

Fig. 3. (a) CIE coordinates of the different colored patterns; (b)-(e) fluorescence images collected from aligned complementary red and green patterns (b) or from superimposed green crosses on red layers with green-to-red concentration ratios per unit area equal to 12 (c), 6 (d) and 3(e). 
$\left(\mathrm{M}_{\mathrm{NCs}}=2 \times 10^{-6} \mathrm{~mol} / \mathrm{l}\right)$ was deposited on a Si substrate and baked at $180^{\circ} \mathrm{C}$ for $2 \mathrm{~min}$. Cross-shaped patterns were realized by means of e-beam lithography, thus locally removing the red emitters. The sample was subsequently cleaved in 3 different parts, and different green emitting NCs/SU-8 blends with (I): $\mathrm{M}_{\mathrm{NCs}}=2 \times 10^{-6} \mathrm{~mol} / \mathrm{l}$, (II): $\mathrm{M}_{\mathrm{NCs}}=4 \times 10^{-6} \mathrm{~mol} / \mathrm{l}$ and (III): $\mathrm{M}_{\mathrm{NCs}}=8 \times 10^{-6} \mathrm{~mol} / \mathrm{l}$, respectively, were spin-coated on top of them. The deposition parameters were chosen in order to obtain a SU-8 layer $\sim 3$ times higher than the PMMA one. By taking into account the ratios between the thicknesses of the layers, the green-to-red molar ratio per unit area $\mathrm{C}_{\text {green }} / \mathrm{C}_{\text {red }}$ is about 3, 6 and 12 for samples (I), (II) and (III), respectively. Complementary cross patterns were realized through photolithography. After development, PL spectra were detected by means of the Fluoview confocal system coupled with a photodetector through a monochromator; fluorescence images of the different patterns were also collected by coupling the microscope with a color CCD camera. The CIE coordinates of the emitted light were calculated and reported in the CIE diagram in Fig. 3a. Insets show fluorescence images collected from a region where a green cross was aligned to a complementary cross-shaped hole in the red layer (Fig. 3b) or from a superimposed cross of (III) NCs/SU-8 blend (Fig. 3c), (II) NCs/SU-8 blend (Fig. 3d) and (I) NCs/SU-8 blend (Fig. 3e) on a uniform red layer. As it is evident from the CIE diagram, the emission detected from superimposed patterns is a linear combination of red and green NCs emission, corresponding respectively to the black circle and black triangle points. By increasing the green-to-red molar concentration ratio per unit area, the corresponding CIE coordinates shift from the red towards the green region, turning to a yellowish color when $C_{\text {green }} / C_{\text {red }}$ is $\sim 12$. Since the NCs extinction coefficient at $405 \mathrm{~nm}$ decreases as the NCs size decreases, we can expect that a higher concentration of the smaller green emitting Quantum Dots is required in order to appreciably modify the overall pattern color. Moreover, we observed in Fig. 3e that the main effect of the low-concentration $\mathrm{NCs} / \mathrm{SU}-8$ blend on the sample is just darkening of the underlying red emission; this is mostly due to absorption of the excitation wavelength by means of both SU- 8 and green NCs, while the green emission itself is still too weak to be distinguished by human eye.

We have thus demonstrated the possibility to realize multicolored pattern by exploiting precise localization of colloidal nanocrystals dispersed in polymeric blends sensitive to electron beam or ultraviolet radiation. This technology has been actually verified with red and green emitting Quantum Dots, but it can be also extended to blue emitters. In this way, the full NTSC (National Television System Committee) color range can be covered by exploiting just three NCs ensembles, and white-emitting pixels can be easily obtained.

Moreover, the same resolution presently reached by photolithographic techniques on industrial scale (standard $0.25 \mu \mathrm{m}$ CMOS technology for VLSI circuits) allows the localization of resist-based
RGB pixels smaller than $1 \mu \mathrm{m}^{2}$, well below the minimum dimensions recognized by the human eye. This means that our approach could be directly transferred to mass production to obtain very high definition color images at very low-costs. Further studies will also verify the possibility to combine our technology with electrical injection already proposed for Quantum Dots based single-colored LEDs [21], thus allowing the realization of bright, stable and cheap high resolution multicolor displays, for the next generation of video systems.

\section{Acknowledgements}

The authors gratefully acknowledge dott. Liberato Manna and dott. Luigi Carbone for the synthesis of colloidal nanocrystals, dott. Milena De Giorgi and dott. Giovanni Morello for assistance in optical characterization, and dott. Marco Mazzeo for useful discussions. The expert technical help of Gianmichele Epifani and Paolo Cazzato is also recognized.

\section{References}

[1] B.W.D'. Andrade, M.E. Thompson, S.R. Forrest, Adv. Mater. 14 (2002) 147.

[2] X.H. Zhang, M.W. Liu, O.Y. Wong, C.S. Lee, H.L. Kwong, S.T. Lee, S.K. Wu, Chem. Phys. Lett. 369 (2003) 478.

[3] C.D. Muller, A. Falcou, N. Reckefuss, M. Rojahn, V. Wiederhim, P. Rudati, H. Frohne, O. Nuyken, H. Becker, K. Meerholz, Nature 421 (2003) 829.

[4] J.W. Orton, C.T. Foxon, Rep. Prog. Phys. 61 (1998) 1.

[5] M. Granström, O. Inganäs, Appl. Phys. Lett. 68 (1996) 147.

[6] R.S. Deshpande, V. Bulovic, S.R. Forrest, Appl. Phys. Lett. 75 (1999) 888.

[7] G. Li, J. Shinar, Appl. Phys. Lett. 83 (2003) 5359.

[8] See, for example, OLED televisions on SONY website.

[9] A.P. Alivisatos, Science 271 (1996) 933.

[10] L. Manna, E.C. Scher, A.P. Alivisatos, J. Am. Chem. Soc. 122 (2000) 12700.

[11] V.I. Klimov, A.A. Mikhailovsky, S. Xu, A. Malko, J.A. Hollingsworth, C.A Leatherdale, H.J. Eisler, M.G. Bawendi, Science 290 (2000) 314

[12] B. Mahler, P. Spinicelli, S. Buil, X. Quelin, J.-P. Hermier, B. Dubertret, Nature Mater. 7 (2008) 659.

[13] M.C. Schlamp, X.G. Peng, A.P. Alivisatos, J. Appl. Phys. 82 (1997) 5837.

[14] H. Yang, P.H. Holloway, J. Phys. Chem. B 107 (2003) 9705.

[15] S. Coe, W.K. Woo, M. Bawendi, V. Bulovic, Nature 420 (2002) 800.

[16] Y.Q. Li, A. Rizzo, M. Mazzeo, L. Carbone, L. Manna, R. Cingolani, G. Gigli, J. Appl. Phys. 97 (2005) 113501

[17] L. Pang, Y. Shen, K. Tetz, Y. Fainman, Opt. Expr. 13 (2005) 44

[18] L. Martiradonna, T. Stomeo, L. Carbone, G. Morello, A. Salhi, M. De Giorgi, R. Cingolani, M. De Vittorio, Phys. Stat. Sol. 243 (15) (2006) 3972.

[19] A. Rizzo, M. Mazzeo, M. Palumbo, G. Lerario, S.D'. Amone, R. Cingolani, G. Gigli, Adv. Mater. 20 (2008) 1886.

[20] S. Jun, E. Jang, J. Park, J. Kim, Langmuir 22 (2005) 2407.

[21] Q. Sun, Y.A. Wang, L.S. Li, D. Wang, T. Zhu, J. Xu, C. Tang, Y. Li, Nature Phot. 1 (2007) 717.

[22] PMMA-950K positive electron beam resist, from MicroChem Corp.

[23] SU-8 2002 negative photo and electron-beam resist, from MicroChem Corp.

[24] P. Reiss, J. Bleuse, A. Pron, Nanoletters 7 (2002) 781

[25] B.O. Dabboussi, J. Rodriguez-Viejo, F.V. Mikulec, J.R. Heine, H. Mattoussi, R. Ober, K.F. Jensen, M.G. Bawendi, J. Phys. Chem. B 101 (1997) 9463.

[26] A. Arceo, L. Meli, P.F. Green, Nanoletters 8 (2008) 2271.

[27] L. Martiradonna, A. Qualtieri, T. Stomeo, L. Carbone, R. Cingolani, M. De Vittorio, Sensors and Actuators B 126 (2007) 116. 